\title{
Preoperative biomechanical mapping of the female pelvic floor
}

\author{
Lennox Hoyte ${ }^{1}$ and Vladimir Egorov ${ }^{2 *}$ \\ ${ }^{1}$ The Pelvic Floor Institute, Tampa, Florida, USA \\ ${ }^{2}$ Artann Laboratories, Trenton, New Jersey, USA
}

\section{Background}

Many of female pelvic floor disorders, including pelvic organ prolapse (POP), stress urinary incontinence, sexual dysfunction, congenital anomalies and others, are clearly manifested in the mechanical properties of pelvic organs. Therefore, biomechanical mapping of a response to applied pressure or load within the pelvic floor opens new possibilities in biomechanical assessment and monitoring of pelvic floor conditions. An innovative approach, vaginal tactile imaging, allows biomechanical mapping of the female pelvic floor including assessment of tissue elasticity, pelvic support and pelvic muscle functions in high definition [1-3]. This case report on a patient with POP demonstrates preoperative tactile imaging, functional tactile imaging and brings an example of the biomechanical parameters (vaginal tissue elasticity, support structures and functions) of the pelvic floor. This allows identification of pelvic structures contributing into the POP development for the patient.

\section{Case report}

We report here on 68-year-old patient who complained of increasing vaginal pressure, discomfort, backache and bulging exacerbated by lifting and straining. A standard physical examination was performed including a bimanual pelvic examination and Pelvic Organ Prolapse Quantification (POP-Q) [4]. The pelvic floor conditions were categorized as POP Stage III in anterior and POP Stage II in posterior compartments; no uterine prolapsed and no prior pelvic surgery. The patient had stress urinary incontinence. To identify pelvic structures contributing into the POP development, the FDA approved device, Vaginal Tactile Imager (VTI), model $2 S$ (Advanced Tactile Imaging, Trenton, NJ) was used in this case. The VTI examination procedure according to CPT Code 0487T (biomechanical mapping, transvaginal, with report) included 8 tests: (1) probe insertion, (2) elevation, (3) rotation, (4) Valsalva maneuver, (5) - (6) voluntary muscle contractions, (7) involuntary relaxation, and (8) reflex contraction (cough). The VTI test results are present in Figures 1-8.

\section{Discussion}

The VTI Tests 1 and 3 provide data related to the vaginal tissue elasticity (Figures 1 and 3 ). Test 2 provides identified parameters (pressure responses to tissue deflection) related to the pelvic support structure (Figure 2). Tests $4-8$ provide comprehensive information about the pelvic functions such as muscles contraction strength and muscle mobility (Figures $4-8$ ). The following surgical procedures were completed on this patient to correct the pelvic deficiency: sacral colpopexy; abdominal enterocele repair and sling insertion.

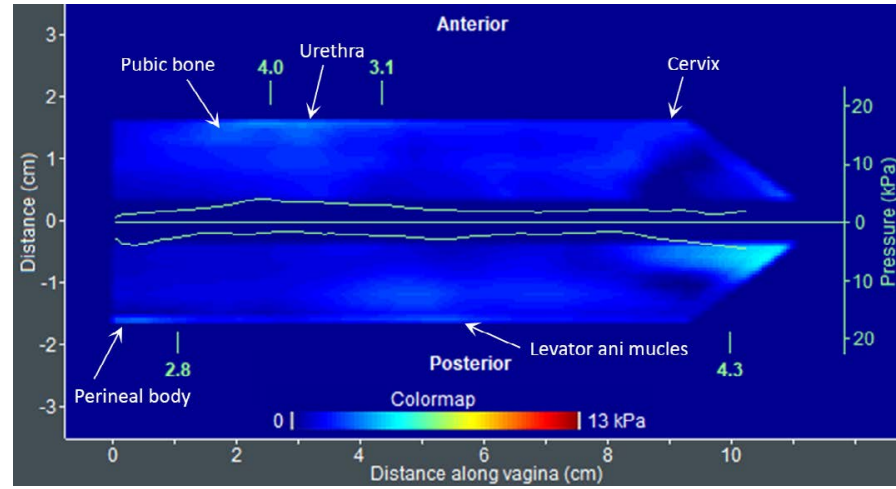

Figure 1. A tactile image acquired during the VTI probe insertion (Test 1) with anatomical landmarks and maximum pressure graphs (green lines, $\mathrm{kPa}$ ) along anterior and posterior compartments

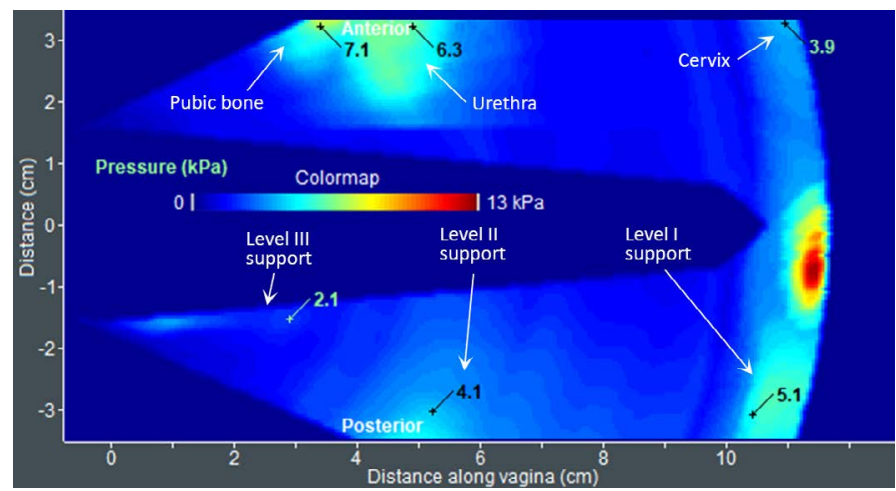

Figure 2. A tactile image acquired during the VTI probe elevation (Test 2) with anatomical landmarks and pressure values at specified locations

${ }^{\star}$ Correspondence to: Vladimir Egorov, Artann Laboratories, 1457 Lower Ferry Rd, Trenton, NJ, USA, E-mail: vti@tactile-imaging.com

Key words: female pelvic floor, prolapse, biomechanical matting, tactile imaging, tissue elasticity, support structures, pelvic function

Received: August 03, 2018; Accepted: August 20, 2018; Published: August 22, 2018 


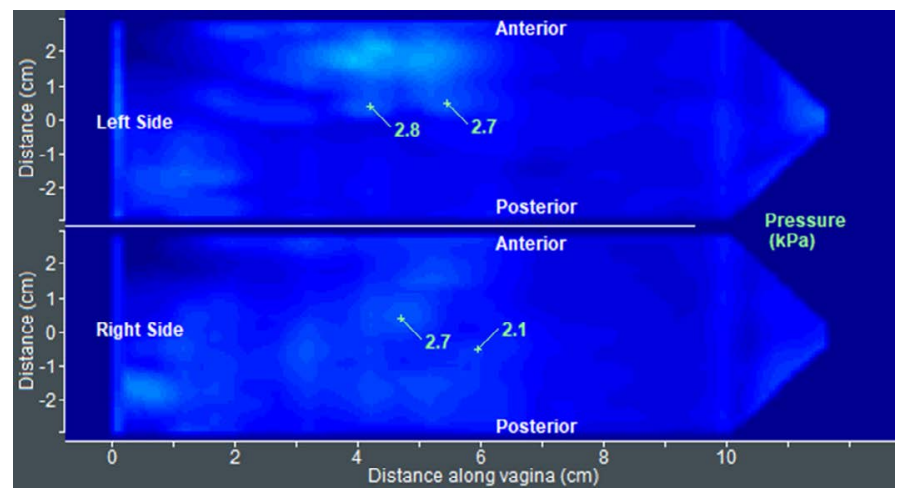

Figure 3. A tactile image acquired during the VTI probe rotation (Test 3) with pressure values at specified locations

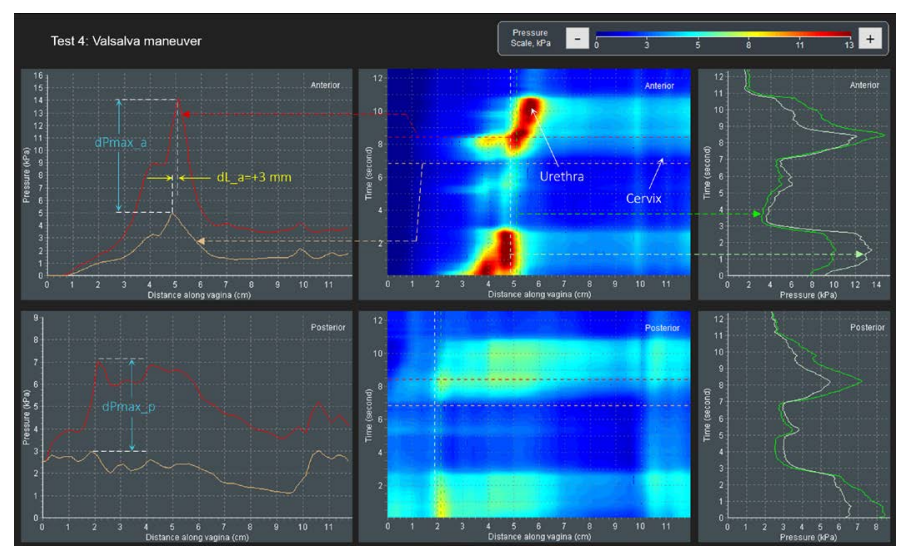

Figure 4. A dynamic pressure patterns acquired during the Valsalva maneuver for anterior and posterior compartments (Test 4)

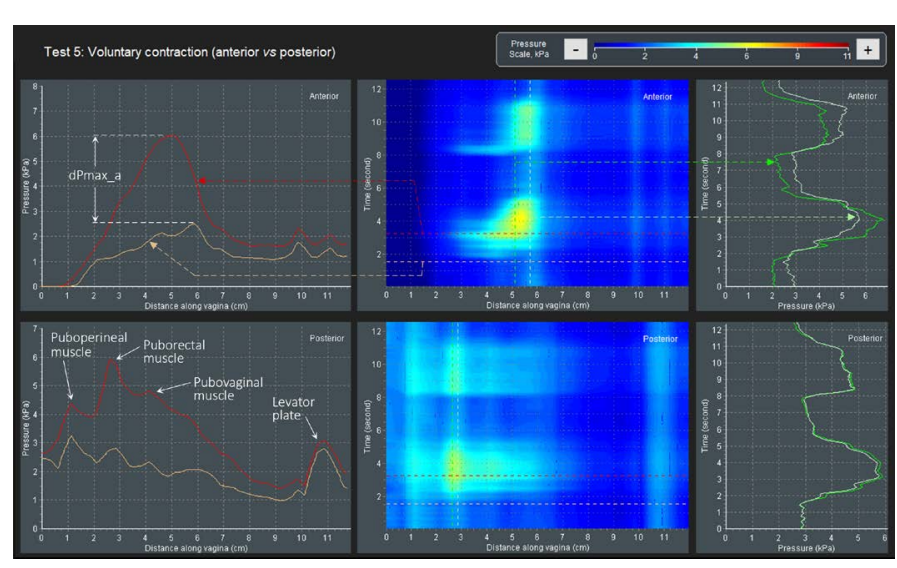

Figure 5. A dynamic pressure patterns acquired during the voluntary muscle contraction for anterior and posterior compartments (Test 5)

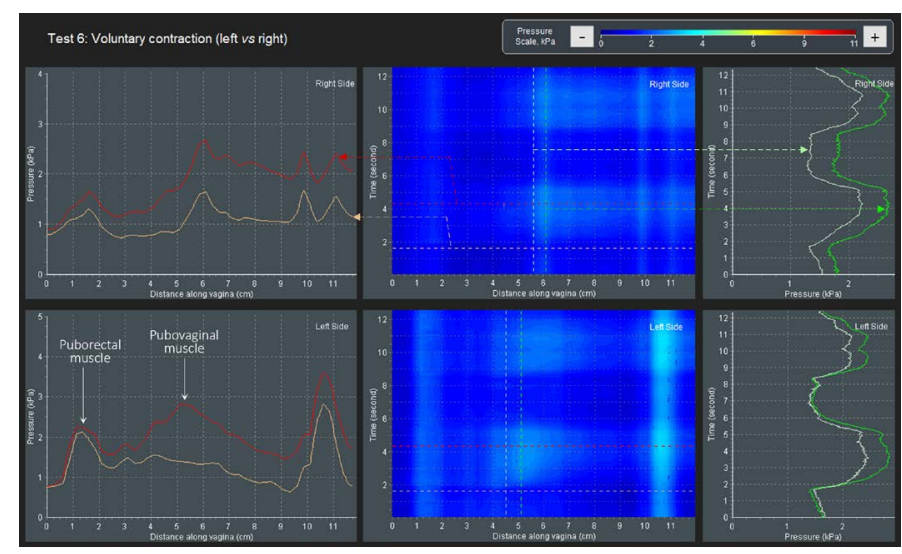

Figure 6. A dynamic pressure patterns acquired during the voluntary muscle contraction for left and right vaginal compartments (Test 6)

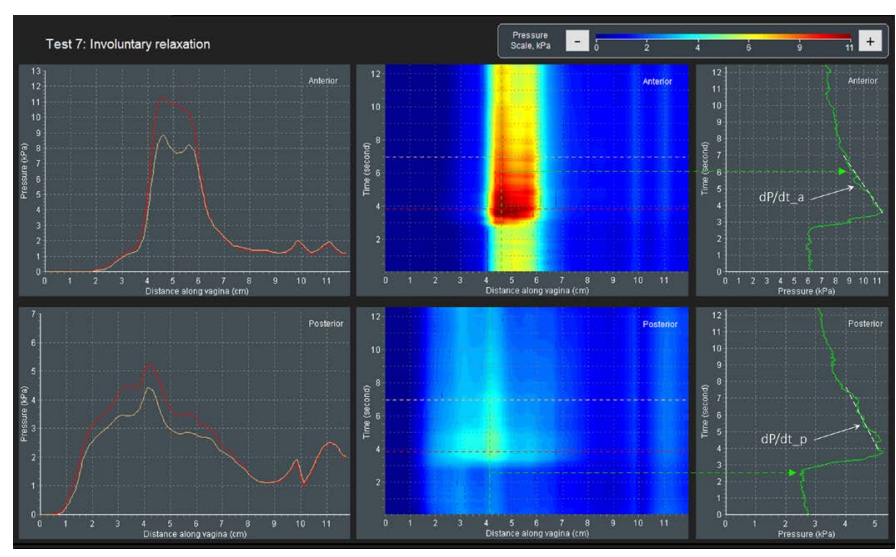

Figure 7. A dynamic pressure relaxation acquired during the involuntary muscle relaxation for interior and posterior compartments (Test 7)

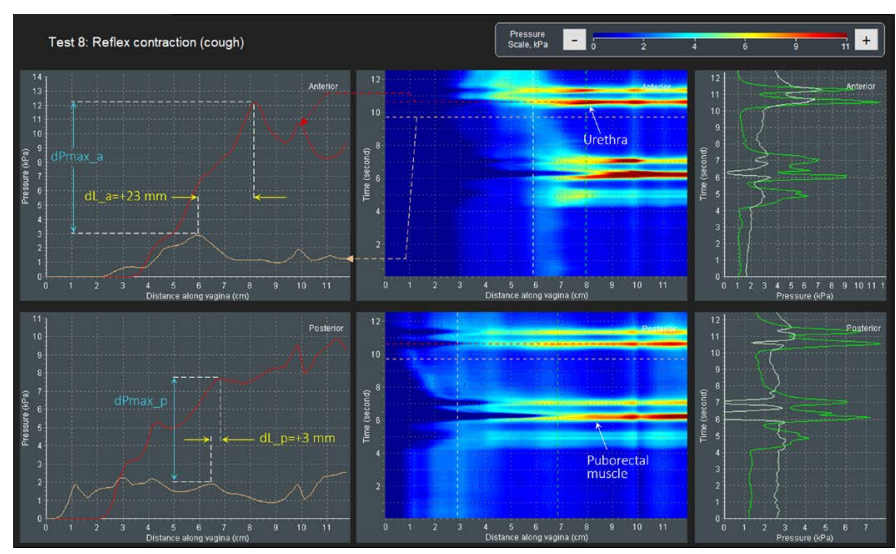

Figure 8. A dynamic pressure patterns acquired during the reflex contraction (cough) for anterior and posterior compartments (Test 8) 


\section{Conclusions}

The biomechanical mapping of the female pelvic floor allows comprehensive data for tissue elasticity, pelvic support and function. The VTI may be a useful preoperative imaging tool.

\section{Acknowledgements}

Research reported in this publication was supported by the National Institute on Aging of the National Institutes of Health under Awards Number SB1AG034714. The content is solely the responsibility of the authors and does not necessarily represent the official views of the National Institutes of Health.

\section{Disclosure}

L. Hoyte has no conflicts of interests. V. Egorov is a CEO and shareholder of Advanced Tactile Imaging, Inc.

\section{References}

1. Egorov V, van Raalte H, Lucente V, Sarvazyan A (2016) Biomechanical characterization of the pelvic floor using tactile imaging. In: Biomechanics of the Female Pelvic Floor, Eds. Hoyte L, Damaser MS, 1st Edition, Elsevier: 317-348.

2. Kim K, Egorov V, Shobeiri SA (2017) Emerging imaging technologies and techniques In: Practical Pelvic Floor Ultrasonography, Ed. Abbas Shobeiri, 2nd Edition, Springer International Publishing AG: 327-336. 3.

3. Lucente V, van Raalte H, Murphy M, Egorov V (2017) Biomechanical paradigm and interpretation of female pelvic floor conditions be-fore a treatment. Int $J$ Womens Health 9: 521-550. 4. [Crossref]

4. Bump RC, Mattiasson A, Bø K, Brubaker LP, DeLancey JO, et al. (1996) The standardization of terminology of female pelvic organ prolapse and pelvic floor dysfunction. Am J Obstet Gynecol 175: 10-17. [Crossref]

Copyright: (2018 Hoyte L. This is an open-access article distributed under the terms of the Creative Commons Attribution License, which permits unrestricted use, distribution, and reproduction in any medium, provided the original author and source are credited. 